Anjou. Maine. Poitou-Charente. Touraine

108-4 | 2001

La Chambre des comptes de Bretagne

\title{
Un magistrat consciencieux de la Chambre des comptes de Bretagne Salomon de La Tullaye (1599-1675)
}

Héloïse Menard

\section{(2) OpenEdition \\ Journals}

Édition électronique

URL : http://journals.openedition.org/abpo/1690

DOI : $10.4000 /$ abpo. 1690

ISBN : 978-2-7535-1484-3

ISSN : $2108-6443$

\section{Éditeur}

Presses universitaires de Rennes

\section{Édition imprimée}

Date de publication : 20 décembre 2001

Pagination : 249-274

ISBN : 978-2-86847-674-6

ISSN : 0399-0826

\section{Référence électronique}

Héloïse Menard, « Un magistrat consciencieux de la Chambre des comptes de Bretagne Salomon de La Tullaye (1599-1675) », Annales de Bretagne et des Pays de l'Ouest [En ligne], 108-4 | 2001, mis en ligne le 20 décembre 2003, consulté le 23 avril 2019. URL : http://journals.openedition.org/abpo/1690 ; DOI : 10.4000/abpo.1690 


\title{
Un magistrat consciencieux de la Chambre des comptes de Bretagne Salomon de La Tullaye (1599-1675)
}

\author{
HéloÏse MENARD ${ }^{1}$
}

La famille de La Tullaye, issue de la petite et ancienne noblesse bretonne, accède à la Chambre des comptes de Bretagne au XVI ${ }^{\mathrm{e}}$ siècle, avec la nomination de Yves de La Tullaye à la charge de secrétaire, auditeur et greffier aux comptes en 1555. Trois de ses fils lui succèdent à la Chambre, Alexandre et Jean devenant à leur tour auditeurs puis maîtres aux comptes, André avocat général. L'épisode de la Ligue bretonne, à l'extrême fin du XVI ${ }^{\mathrm{e}}$ siècle, constitue une heureuse opportunité pour la famille, qui peut nouer d'intéressantes alliances matrimoniales avec des lignages en vue comme les Bitault ou les Fourché.

Le XVII ${ }^{\mathrm{e}}$ siècle est celui de l'ascension : René de La Tullaye devient maire de la ville de Nantes. C'est son cousin ${ }^{2}$ Salomon de La Tullaye qui recueille à sa mort, en 1635, son office de maître à la Chambre des comptes, ainsi que la tutelle de ses deux fils mineurs. Pour garder une trace tant de ses activités à la Chambre que de ses dépenses domestiques, Salomon de La Tullaye entreprend la rédaction d'un livre de raison, sous forme de plusieurs cahiers de petite taille. C'est ce document, conservé comme beaucoup d'autres dans le vaste fonds La Tullaye aux Archives Départementales de Loire-Atlantique, qui a permis une première étude de cette famille de la noblesse nantaise, et plus particulièrement de son auteur, Salomon de La Tullaye, sieur de la Suze, du Plessis-Tison et d'autres domaines de la région nantaise.

Après avoir présenté le personnage et son lieu de vie, nous expliquerons pourquoi Salomon de La Tullaye a rédigé un livre de raison. Viendra ensuite un développement sur l'office de maître aux comptes exercé par Salomon de La Tullaye à la Chambre des comptes de Bretagne. La vie quo-

1. Auteur d'un mémoire de maîtrise intitulé Ascension politique et sociale de la famille de La Tullaye, $X V{ }^{e}-\mathrm{XVI} \mathrm{I}^{\mathrm{s}}$ siècles, réalisé sous la direction de Dominique Le Page, Université de Nantes, 2000, 163 p.

2. Cf. tableau généalogique simplifié de la famille de La Tullaye aux $\mathrm{XVI}^{\mathrm{e}}-\mathrm{XVII}{ }^{\mathrm{e}}$ siècles (annexe 1) 
tidienne et les relations familiales, vues à travers le prisme des livres de raison, feront l'objet d'une dernière partie.

\section{Un homme d'importance qui tient son rang dans la cité}

\section{Salomon et ses héritages}

Son père, Charles de La Tullaye, sieur du Mée, a épousé en 1595 Françoise de Vaucouleurs, fille d'un seigneur de Dinan. De cette union sont nés six enfants, mais seuls deux d'entre eux ont survécu : Salomon et Marquise. Le petit Salomon est baptisé le 18 juillet $1599^{3}$ et doit son prénom à son parrain Salomon de Herbammez, archidiacre de la Mée et official de Nantes. Après la mort de Charles de La Tullaye en avril 1607, Françoise de Vaucouleurs se remarie, à Saint Donatien de Nantes, avec François de Cadaran, seigneur du Plessis-Tison, dont elle n'a qu'une fille : Françoise de Cadaran. Lorsqu'elle se trouve veuve pour la seconde fois, la mère de Salomon se démet de tous ses biens entre les mains de son seul fils - au grand dam de la jeune Françoise de Cadaran, qui se voit ainsi dépouillée des biens de son père - en $1635^{4}$.

C'est ainsi que Salomon de La Tullaye se retrouve à la tête d'une fortune non négligeable, car sa mère, Françoise de Vaucouleurs, est l'unique héritière de quatre familles ${ }^{5}$. L'héritage des familles Blanchet et Tempéran apporte à Salomon les terres de la Tour et de la Guilbaudière, à HauteGoulaine, au sud de Nantes, tandis que les biens des Le Roy se composent de domaines à Rome. Thomas le Roy (qui avait latinisé son nom en Thomas Regis) y exerçait de nombreuses charges honorifiques, parmi lesquelles celle de " procureur du dernier concile général de Latran nommé par le pape Jules II ${ }^{6}$ " en 1515 , avant de devenir " clerc de la chambre apostolique, emploi des plus considérables de la magistrature romaine ". La mort sans héritier de tous ses frères et neveux fait passer cet important héritage des Le Roy aux La Tullaye, notamment une chapelle construite par Thomas Regis dans la collégiale Notre-Dame de Nantes. Cette chapelle que les La Tullaye conserveront et prendront soin d'entretenir, contient d'ailleurs plusieurs enfeux de la famille ${ }^{7}$. À ceci s'ajoute l'héritage du beau-père de Salomon, François de Cadaran, qui lui offre le château du Plessis-Tison, à

3. Arch. dép. de Loire-Atlantique, 2 E 1383/2, $1^{\text {re }}$ liasse : copie du baptême certifiée conforme à la minute le 16 avril 1788 .

4. Bibliothèque nationale, Carré de d'Hozier-Tugnot 614 : acte de démission de Françoise de Vaucouleurs entre les mains de son fils Salomon, reçu par Desmortiers, notaire à Nantes, le 11 septembre 1635 .

5. Cf. arbre généalogique des familles Le Roy, Blanchet, Tempéran, Vaucouleurs et La Tullaye (Annexe 4)

6. Stéphane de La Nicolliere, Église royale et collégiale de Notre-Dame de Nantes, Nantes/Paris, Forest-Grimaud/Aubry, 1865, p. 114-120.

7. Arch. dép. de Loire-Atlantique, 23 Fi Nantes n 1216 et n 1217 : quelques cartes postales de R. Guénault reproduisant des gravures anciennes montrent l'intérieur et l'extérieur de la chapelle Saint Thomas avant la destruction de la collégiale Notre-Dame au XIX ${ }^{\mathrm{e}}$ siècle. 
Nantes $^{8}$. Cependant, ce n'est pas dans ce château que Salomon de La Tullaye va s'établir, mais à l'Hôtel de la Suze, en plein cœur de Nantes. L'histoire de cet hôtel est suffisamment curieuse pour mériter que l'on s'y arrête un instant.

\section{Figure $n^{\circ} 1$ - Le château du Plessis-Tison}

327. Châteaux de la Loire-Inférieure - NANTES - Château du Plessis-Tison (Façade sud)

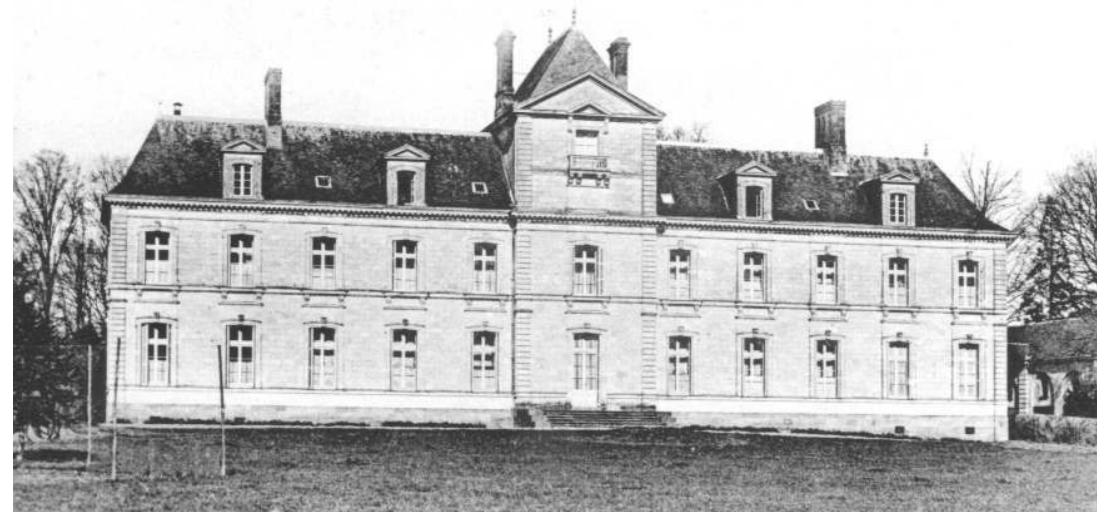

\section{Salomon et l'hôtel de la Suze}

Situé à deux pas de la cathédrale de Nantes, ce vieux manoir aujourd'hui disparu, traîne derrière lui un passé au parfum de soufre, lié à Gilles de Rais. Le nom de la Suze est d'ailleurs celui de l'une des seigneuries de la famille de Rais, près de Sablé. Après avoir été dûment exorcisé et rebaptisé hôtel de Montfort, le bâtiment passe aux mains du dernier duc de Bretagne, François II, qui en fait une résidence ducale. L'hôtel devient ensuite propriété de Charles VIII et d'Anne de Bretagne, qui veulent y installer, en 1495, la Chambre des comptes de Bretagne. Le refus des gens des comptes, qui lui préfèrent le couvent des Cordeliers ${ }^{9}$, plonge l'hôtel dans l'abandon. Ce sont les héritiers de la succession de Rais (ou Retz) qui finissent par vendre en 1543 cette " maison ruineuse ${ }^{10}$ " à Françoise Le Frère. Cette dernière, fille de Charles le Frère et de Jeanne Censier ${ }^{11}$ (veuve de Nicolas de La

8. Le château du Plessis-Tison est actuellement le lycée privé Blanche-de-Castille à Nantes.

9. La Chambre des comptes quitte ensuite le couvent des Cordeliers pour s'installer dans un palais, remplacé au XVIII ${ }^{e}$ siècle par le bâtiment construit par l'architecte Ceineray sur les quais de l'Erdre (actuelle préfecture de Nantes).

10. Arch. dép. de Loire-Atlantique, 2 E 1383/158 : lettres du 18 août 1543 de François I ${ }^{\text {er }}$ à Françoise Le Frère.

11. Cf. arbre généalogique de la famille de La Tullaye (annexes 1, 2 et 3). 
Tullaye) décède sans enfant de ses deux mariages successifs. C'est donc son demi-frère, Yves de La Tullaye (fils du premier mariage de Jeanne Censier avec Nicolas de La Tullaye) qui hérite de la Suze, et la transmet ensuite à ses fils ${ }^{12}$. Un temps revendu à Guillaume Bernard, sieur de Porteric $^{13}$, en 1605, l'hôtel est finalement racheté dès $1606^{14}$ par retrait lignager au nom de Charles de La Tullaye, quatrième fils d'Yves de La Tullaye et père de Salomon, par souci de " conserver ladite maison en leur famille " et ce pour la somme de 12114 livres $^{15}$.

Salomon vient s'établir dans l'hôtel Suze partiellement restauré, en 1636, année de son mariage avec Renée de Lesrat, fille du conseiller au Parlement de Bretagne Guy de Lesrat, originaire d'Angers. Haut de trois ou quatre étages selon les endroits, l'hôtel en tuffeau blanc et recouvert d'ardoises occupe à lui seul le quart de lî̀lot urbain contenu entre les rues Notre-Dame, Saint-Denis et Saint-Vincent, face à la collégiale Notre-Dame ${ }^{16}$ - église où la famille de La Tullaye se fait inhumer. L'emplacement est idéal pour Salomon, à proximité du palais de la Chambre des comptes de Bretagne, situé sur les bords de l'Erdre, en contrebas, institution où il exerce une charge de maître aux comptes. Les restaurations de la Suze et la vie qu'y mène Salomon nous sont connues par le soin qu'il a pris de noter chaque jour ses recettes et dépenses dans ce qu'on appelle les livres de raison. La rareté de ce type d'écrit incite à s'y intéresser de près.

\section{Salomon et les livres de raison}

\section{Qu'est-ce qu'un livre de raison?}

À l'instar de quelques-uns de ses contemporains, Salomon de La Tullaye tient un cahier sur lequel il note toutes ses "recettes et despen$\operatorname{ses}^{17}$ " au jour le jour. Ceci n'est pas nouveau, car dès le Xve siècle, on trouve des diarii en Italie « où des contemporains de toute condition notent plus ou moins exactement, au jour le jour, les faits petits ou grands qui viennent à leur connaissance ${ }^{18}$ ". L'expression " Livre de raison " est utilisée dès l'époque moderne, et le Dictionnaire Universel de Furetières (1690) le définit comme " un livre dans lequel un bon mesnager ou un marchand

\section{Idem.}

13. Arch. dép. de Loire-Atlantique, 2 E 1383/158 : " Estat et enrollement ... " de la Suze.

14. Ibidem, Jean de La Tullaye, neveu de Charles de La Tullaye, reçoit procure de celuici le 26 août 1606, et le retrait lignager de la Suze a lieu le 22 novembre de la même année.

15. Idem, 2 E 1383/158.

16. L'hôtel comme l'église ont malheureusement été détruits au XIX ${ }^{\mathrm{e}}$ siècle, notamment lors des travaux d'aménagement des rues de Nantes et de la percée de la rue de Strasbourg. L'emplacement de la Suze est actuellement occupé par les hôtels Arnous Rivière et de Melient.

17. Arch. dép. de Loire-Atlantique, 2 E 1383/12 : livre de raison de Salomon de La Tullaye.

18. H. HAUSER, Sources de l'Histoire de France, tome 1, p. 10, cité par Élisabeth BourCIER, Les journaux privés en Angleterre de 1600 à 1660, Publications de la Sorbonne, Imprimerie Nationale, Paris, 1976, p. 16. 


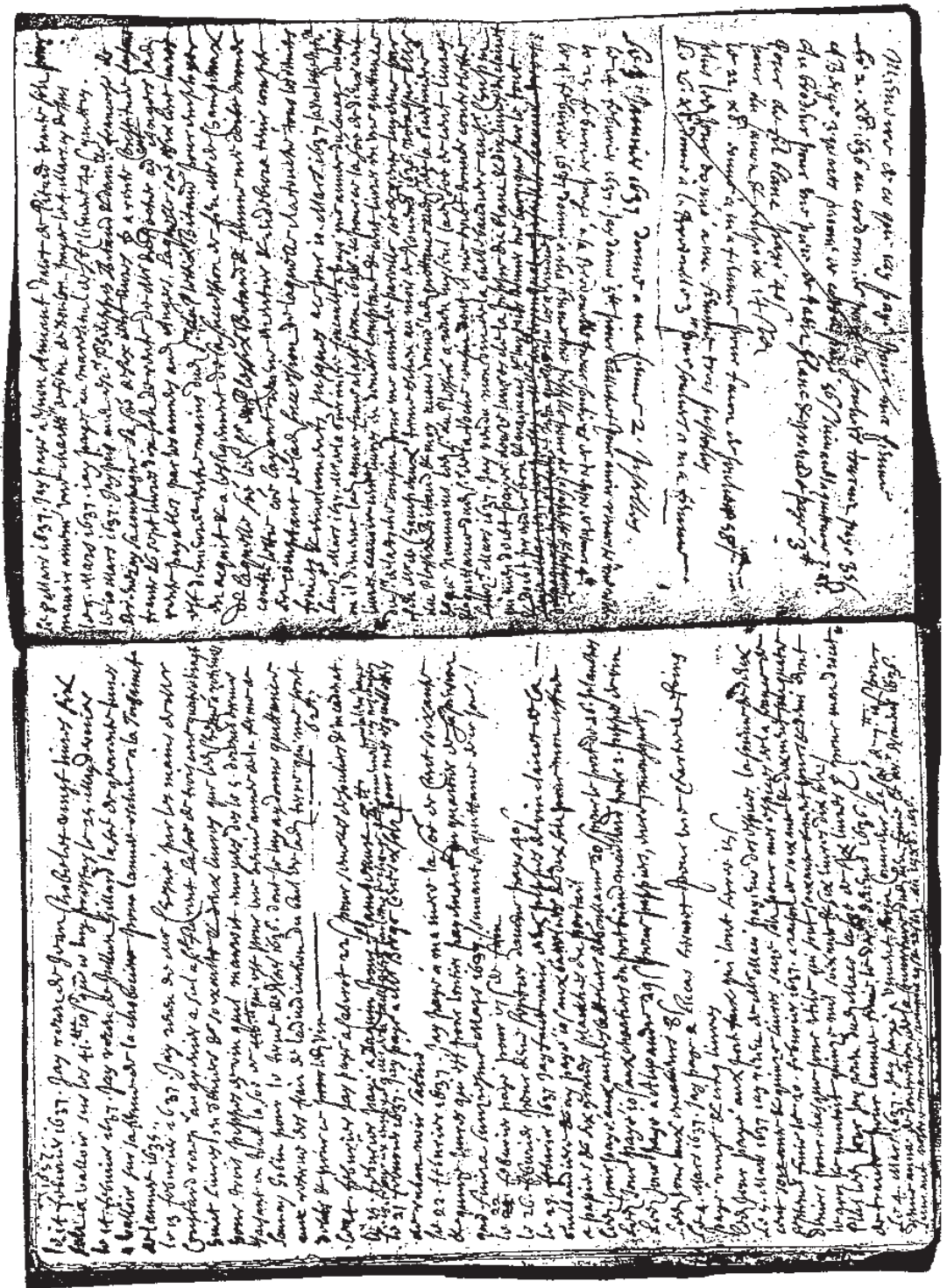

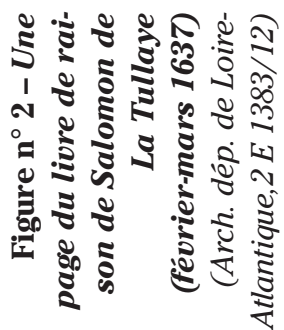


escrit tout ce qu'il reçoit et despense, pour se rendre compte et à raison à luy mesme de toutes ses affaires ". Ceci correspond parfaitement aux écrits de Salomon de La Tullaye, qui, pour n'être pas marchand, n'en est pas moins un très "bon mesnager ", soucieux de ses deniers! Les travaux d'Élisabeth Bourcier sur les journaux anglais du XVII ${ }^{\mathrm{e}}$ siècle affinent encore la définition. L'auteur du livre de comptes le rédige à la première personne, dans un style sec et répétitif, sans aucune recherche littéraire, et où l'unité de temps n'excède jamais la journée. Ainsi Salomon écrit-il le plus souvent " j'ay receu " et "j'ay payé ${ }^{19}$ ", les paiements concernant généralement les frais de la vie quotidienne, tandis les fermages forment le gros des recettes. De la quotidienneté de cette rédaction vient le mot diaire ou dial en français, diary en anglais, termes issus du latin dies : le jour. Salomon intitule lui-même ses cahiers "Dial de mes affaires domestiques ${ }^{20}$ ". Le but de ces cahiers est ici comptable, on n'y trouve pas de sentiments ni de pensées, au contraire de certains livres de raison, comme celui, truculent, de Gilles de Gouberville au siècle précédent ${ }^{21}$.

L'exceptionnelle durée de la rédaction, qui approche les quarante ans, compense cependant la sécheresse du style. En effet, Salomon commence à écrire en 1636, et s'arrête en septembre 1674, il a alors 75 ans, et l'heure de la mort approche ${ }^{22}$. Bien qu'âgé, Salomon garde une écriture aussi lisible que les années précédentes, et l'absence de tout apparat littéraire ne saurait faire oublier la netteté de sa calligraphie et le peu de fautes d'orthographe commises, ce qui n'est pas le cas de nombreux autres écrits de son époque.

\section{Pourquoi commence-t-il à écrire? Les comptes de tutelle}

La première raison motivant cette rédaction semble être d'ordre familial. En effet, en 1635, Salomon devient, suite au décès de son cousin René de La Tullaye ${ }^{23}$, tuteur de ses deux fils mineurs René et Jacques, âgés respectivement de dix-huit et de quatorze ans. La responsabilité de la tutelle implique la tenue de comptes nets, que le tuteur devra ensuite pré-

19. Arch. dép. de Loire-Atlantique, 2 E 1383/12 : livre de raison de Salomon de La Tullaye. 20. Ibidem.

21. Madeleine FoIsI, Le Sire de Gouberville, un gentilhomme normand au XVI siècle, Aubier, Paris, 1981.

22. La date exacte de la mort de Salomon est inconnue. Dans son Dictionnaire des magistrats de la Chambre des comptes de Bretagne, Gaëtan d'Aviau de Ternay fixe l'inhumation de Salomon au 17 septembre 1677, mais les registres paroissiaux de l'église Notre-Dame de Nantes ne font état d'aucun enterrement à cette date. Les recherches dans les registres paroissiaux des églises Notre-Dame, Saint-Denis et Saint-Donatien, pour les années 1674 à 1677 ne mentionnent pas non plus cette inhumation. Le seul indice provient d'un acte du 7 mai 1675 au siège de la sénéchaussée d'Anjou, concernant Françoise Martineau, seconde femme de Salomon, en tant que veuve (Arch. dép. de Loire-Atlantique, $2 \mathrm{E}$ 1383/7). Ceci placerait la mort de Salomon entre septembre 1674 et mai 1675.

23. Arch. dép. de Loire-Atlantique, 2 E 1383/10 : René de La Tullaye décède le 10 février 1635. 
senter, à la majorité des deux garçons, devant le sénéchal de Nantes. Salomon se lance alors dans la rédaction d'un grand cahier, intitulé "Papier de recepte et de despenses que j'ay faicte en qualité de tuteur des enfans mineurs de feu René de La Tullaye ${ }^{24}$ ". Dans ce cahier, il inscrit ses comptes de tutelle, tant les dépenses qu'il engage pour les deux garçons, que les revenus qu'il perçoit en leur nom. Le cahier est divisé en deux : un côté pour chaque garçon. Le côté réservé à l'aîné, René, est plus important, car Salomon y note, outre les dépenses courantes pour son entretien et son éducation, les revenus des terres et domaines qui lui reviennent en sa qualité d'aîné. En effet, parmi les autres enfants de feu René de La Tullaye, sur les six premiers, seuls deux sont parvenus à l'âge adulte, mais sont décédés en 1635, la même année que leur père. Il reste alors deux filles vivantes, l'une au couvent, et la plus jeune, Louise, mariée avant la mort de son père, et ne dépendant donc pas de la tutelle de Salomon. Le jeune René de La Tullaye se trouve ainsi être l'aîné de la famille et propriétaire de la seigneurie de Belle Isle appartenant à son défunt père.

C'est vraisemblablement à cette occasion que Salomon entreprend de tenir de même ses propres comptes domestiques, mais sur des cahiers cousus d'un format beaucoup plus petit - une feuille pliée en huit. Les années 1635-1636 sont en effet une période de grands changements pour lui : avant même d'être marié - il épouse Renée de Lesrat en 1636 - il est tuteur de deux adolescents. Ces derniers sont envoyés au très réputé Collège Royal de la Flèche ${ }^{25}$, en Anjou, pour y terminer leurs études. Bien que d'ancienne noblesse ${ }^{26}$, les La Tullaye ne dédaignent pas l'instruction comme tant d'autres familles nobles de Bretagne. D'après les comptes de Salomon, une année de pension à la Flèche pour un élève coûte 204 livres, auxquelles il faut ajouter un habit neuf de cinquante livres chaque trimestre, des "menues fournitures ${ }^{27}$ ", les gages des serviteurs, de l'argent de poche. Au total, le montant atteint cinq cent livres, soit un millier de livres pour les deux garçons. René ne restera que deux ans à la Flèche, de 1635 à 1637, contre sept ans pour son frère Jacques, de 1636 à 1643. Une fois émancipés, tous deux s'engagent dans l'armée, René comme « enseigne de compagnie dans la garnison de Moyennie ${ }^{28}$ " (actuelle Moyenneville, arrondissement d'Arras) puis commandant " de l'enseigne colonnelle du régiment de Bourdonné ", et son cadet Jacques " capitaine au régiment de $\mathrm{Mr}$ du Bosquet " en Flandre. La France est alors en guerre contre l'Espagne, dans le cadre de la terrible guerre de Trente Ans, et c'est à la frontière nord de la France que l'on se bat. Les deux jeunes gens laissent à leur tuteur le

\section{Ibidem.}

25. Fondé par Henri IV en 1603 et confié aux jésuites, c'est l'un des plus prestigieux pensionnats de province.

26. Arch. dép. de Loire-Atlantique, 2 E 1383/2 : actes des 13 et 19 octobre 1668 confirmant les La Tullaye dans leur noblesse et leur titre d'écuyer, sur la base de preuves remontant au début du XVe siècle.

27. Idem, 2 E $1383 / 10$.

28. Ibidem. 
soin de continuer à gérer leurs affaires en leur absence, la probité de celuici les satisfaisant visiblement pleinement.

Salomon a également une seconde raison de commencer à écrire : 1636 est également la date de son entrée à la Chambre des comptes de Bretagne.

\section{Salomon, maître à la Chambre des comptes}

\section{L'adjudication de l'office}

Salomon devient en 1636 titulaire de l'office de maître aux comptes exercé par son défunt cousin, dont il a racheté l'office pour une somme de 47000 livres. Le paiement de l'office et les revenus qu'il en tire sont à l'origine d'un nouveau cahier : le «Papier dial du revenu de [s]on office de maistre des comptes en Bretagne ${ }^{29}$ ». On y apprend que c'est en tant que tuteur, élu par le conseil de famille le 9 mars $1635^{30}$, que Salomon a été chargé de procéder à l'adjudication de cet office dont son cousin est décédé revêtu. Cet office, écrit Salomon, " fut adjugé judiciairement à l'audiance du siège présidial de Nantes [...] le $1^{\mathrm{er}}$ mars 1636 au sieur Lorial de Meaux, notaire et secrétaire de la chancellerie de Bretagne à la somme de 47000 livres $^{31}$ ". Mais Salomon peut se féliciter d'avoir pris femme dans un milieu parlementaire et influent, car " l'après disner dudit jour premier mars 1636, les parents de Mr des Briottières - son beau-père, Gui de Lesrat, est seigneur des Briottières - désirèrent que ie demandasse la préférence dudit office et que ie m'y fisse recepvoir ". Jean de Lorial " fist difficultés de le remettre " mais finit par céder, et le nouveau possesseur de l'office conclut : "Sur les cinq heures du soir, ledit sieur de Meaux passa procure au sieur Fouré, procureur au siège présidial de Nantes, rapportée par Quenille, notaire à Nantes, pour consentir le résiliement dudit office et que j'en eusse la préférence ${ }^{32}$. " S'il met en avant la volonté de sa belle-famille dans cette affaire, Salomon montre cependant - à son insu - par l'extrême détail qu'il donne de cette journée, l'importance que cet office revêt pour lui, et nul doute qu'il ne s'en réjouisse grandement!

La vente de sa terre de la Guilbaudière ${ }^{33}$, en 1638, pour 21000 livres, complétée par une partie des 27000 livres de la dot de sa femme, permet à Salomon de rembourser le prix de l'office de maître. Cependant, en 1644, il n'a versé que les deux tiers du total, soit 38489 livres et 16 sols $^{34}$, et se réserve sans doute le droit de continuer à payer au fur et à mesure que ses moyens le lui permettent.

29. Idem, $2 \mathrm{E} 1383 / 17$.

30. Id., $2 \mathrm{E} 1383 / 3$.

31. Id., $2 \mathrm{E} 1383 / 17$.

32. Id., $2 \mathrm{E} 1383 / 17$.

33. Id., 2 E 1383/12 : livre de raison; la Guilbaudière se trouve à Haute-Goulaine, au sud de Nantes. Salomon la vend à Michel Gougeon en 1638.

34. Id., 2 E 1383/3 : état de la première communauté de Salomon et de René de Lesrat. 


\section{Les taxes à payer pour entrer à la Chambre des comptes}

Acheter l'office est une chose, mais entrer à la Chambre des comptes en est une autre. Salomon doit en effet s'acquitter de multiples droits, dûment consignés dans son papier dial habituel, ainsi que dans un papier intitulé "Frais des provisions de mon office de maistre des comptes 35 ". Le sieur de La Tullaye n'est pas avare de papier, et préfère consigner ses affaires en plusieurs exemplaires. Dans le mois suivant l'adjudication de l'office, le 15 avril 1636, 2200 livres sont à payer pour la résignation de l'office à son profit, 324 livres pour le droit de marc d'or, qui est une " sorte de redevance d'honneur ${ }^{36}$ " renouvelable à chaque changement de règne, et 26 livres de frais de sceau. À ceci s'ajoute, le 20 avril, la dispense des quarante jours (puisque le précédent titulaire de la charge est décédé depuis près d'un an), qui se monte à 366 livres et 13 sols, ainsi que le droit annuel de 356 livres, 11 sols et 2 deniers (à payer chaque année pour l'année suivante $^{37}$ ). Salomon peut alors recevoir ses lettres patentes, et il est enfin reçu à la Chambre le 21 mai $1636^{38}$. Le frais ne s'arrêtent cependant pas là, et Salomon écrit : “Le 21 ${ }^{\mathrm{e}}$ May 1636, j'ay presté le serment pour mon office de maistre des comptes, et ledit jour j'ay donné une promesse de 150 escus quart - soit 450 livres - [...] pour payer les droicts de bonnet à Messieurs des comptes ${ }^{39}$. "Au total, son entrée effective à la Chambre lui a coûté 3753 livres, 3 sols et 2 deniers ${ }^{40}$.

\section{Tableau $n^{\circ} 1$ - Taxes acquittées par Salomon de La Tullaye pour entrer à la Chambre des comptes de Bretagne}

\begin{tabular}{|l|r|}
\hline \multicolumn{1}{|c|}{ Taxes } & Montant en livres \\
\hline Pour la résignation & 2200 livres \\
\hline $\begin{array}{c}\text { Pour le marc d'or } \\
\text { (à renouveler à chaque changement de règne) }\end{array}$ & 324 livres \\
\hline Pour l'expédition des lettres de provision & 30 livres \\
\hline Pour le sceau & 26 livres \\
\hline Pour le droit de bonnet & 450 livres \\
\hline $\begin{array}{c}\text { Pour le droit annuel } \\
\text { (à payer chaque année pour l'année suivante) }\end{array}$ & 356 livres, 11 sols et 2 deniers \\
\hline Pour la dispense des 40 jours & 366 livres et 13 sols \\
\hline \multicolumn{1}{c}{ Total } & 3753 livres, 3 sols et 2 deniers \\
\hline
\end{tabular}

Le total n'inclut pas le droit de traite, omis par Salomon.

35. Id., 2 E 1383/21.

36. Mireille JEAN, article " Offices », Dictionnaire de l'Ancien Régime dir. par Lucien Bély, PUF, Paris, 1996, p. 920-923.

37. L'édit de la Paulette, créé en 1604, institue l'hérédité des offices et la liberté pour le titulaire de la transmettre à la personne de son choix, à condition de payer, chaque année et d'avance, une somme correspondant à $1 / 6^{\mathrm{e}}$ de la valeur de l'office.

38. Arch. dép. de Loire-Atlantique, registre B 76, f ${ }^{\circ}$ 285-286 : institution de Salomon de La Tullaye comme maître ordinaire.

39. Idem, 2 E 1383/12 : livre de raison, note du 21 mai 1636.

40. Cf. tableau $\mathrm{n}^{\circ} 1$. 


\section{Le travail d'un maître à la Chambre des comptes}

Durant les vingt années où Salomon va exercer sa charge, entre 1636 et 1656 , sa signature au bas de chaque lettre des mandements royaux ${ }^{41}$ prouve une assiduité jamais démentie. Son travail est essentiellement de procéder, avec les autres maîtres de la Chambre, au jugement des comptes présentés par les officiers comptables de la province, après l'audition réalisée par les auditeurs aux comptes. Il va sans dire que cet office, s'il coûte cher à son propriétaire, lui rapporte cependant des avantages, honorifiques ou plus substantiels. À propos de la vénalité des offices, Roland Mousnier, cite Noël Du Fail s'exclamant qu'il n'y a sujet du roi " tant sot et abesti puisse-t-il estre, qui n'aperçoive au doigt et à l'œil qu'en moins de rien un homme ayant la robe longue [officier de judicature] est riche outre mesure $^{42}$ ! " Mais Du Fail exagère, et les revenus que Salomon tire de son office ne sauraient rembourser les dépenses engagées pour son achat ${ }^{43}$.

\section{Les revenus de l'office de Salomon}

Ce qui apparaît en premier lieu dans le dial domestique de Salomon sont les gages. Ceux-ci restent de 1500 livres durant toute sa carrière, ne représentent que 3 à $4 \%$ du prix de l'office. C'est moins important qu'un revenu foncier. Cependant, aux gages fixes s'ajoutent d'autres petites sommes. Parmi celles-ci, citons les épices, car les officiers " qui exercent également des fonctions judiciaires, perçoivent des droits sur les affaires qui leurs sont soumises". Ainsi " plus un officier travaille, plus il augmente ses revenus $^{44}$ ". Le travail de la Chambre se répartit en deux séances, c'est pourquoi les épices sont payées deux fois par an. En revanche, si le nombre de jours utiles - durant lesquels les officiers travaillent - varie peu, les épices, elles, ne restent pas les mêmes. Ainsi, d'après le dial de Salomon, les 68 jours de la séance de mars 1637 lui rapportent 376 livres, contre 251 livres pour les 66 jours de la séance de septembre $1638^{45}$.

Les « menues nécessitez ", d'une quarantaine de livres en $1639^{46}$, le droit de bonnet ${ }^{47}$, qui est de 6 livres et 8 sols en 1639 et un droit de traite de même valeur, sont autant de petites sommes qui permettent à Salomon d'augmenter ses revenus ${ }^{48}$. La moyenne annuelle des revenus de cet office

41. Arch. dép. de Loire-Atlantique, série B : du registre B 76 au registre B 81.

42. Roland MousNiER, La vénalité des offices sous Henri IV et Louis XIII, PUF, Paris, 1945, éd. revue et augm. 1971, p. 74.

43. Cf. tableau $\mathrm{n}^{\circ} 2$.

44. Olivier Poncet, article « Gages ", Dictionnaire de l'Ancien Régime, op. cit., p. 583-585.

45. Arch. dép. de Loire-Atlantique, 2 E 1383/12 : livre de raison de Salomon.

46. Ibidem, Note du 5 avril 1639 : " J'ay receu de Mr Hervé Bertaud, payeur de nos menues nécessitéz, la somme de 39 livres pour mes menues nécessitéz de 1639."

47. Le droit de bonnet est dû par tout nouvel officier entrant à la Chambre. Salomon a payé le sien, soit 450 livres, le jour de son entrée à la Chambre, les officiers se partageant ensuite la somme.

48. $C f$. tableau $\mathrm{n}^{\circ} 2$. 
n'atteint cependant qu'à peine les 2000 livres. Il faut donc qu'il y ait des avantages autres que pécuniaires à appartenir à la Chambre des comptes. Roland Mousnier souligne que « toute fonction royale [est] d'ailleurs plus ou moins source d'honneurs, que l'on soit officier de finance ou de judicature, grand ou petit officier ${ }^{49} "$. L'un des principaux honneurs consiste en l'anoblissement de l'officier, mais c'est inutile dans le cas de Salomon, qui est d'ancienne famille noble. D'autres avantages sont spécifiés comme " honneurs, authoritéz, franchises, libertéz et immunitéz ${ }^{50}$ " dans les lettres de provision accordées aux officiers, mais ceux-ci n'apparaissent pas dans les écrits de Salomon.

Un tableau des revenus ${ }^{51}$ que Salomon tire de son office de maître des comptes (tableau $\mathrm{n}^{\circ} 2$ ) permet de constater que pour des recettes annuelles de 9200 livres (tableau ${ }^{\circ} 3$ ), les revenus de l'office en question correspondent à environ $10 \%$ du total. Ces derniers, inférieurs à 2000 livres, équivalent à peine à la moitié des revenus fonciers de Salomon (qui s'élèvent à 4200 livres par an) et représentent environ $4 \%$ de sa charge de mâ̂tre des comptes. Tableau $\mathrm{n}^{\circ} 2$ - Revenus de l'office de maître
aux comptes de Salomon de La Tullaye

\begin{tabular}{|l|r|}
\hline \multicolumn{1}{|c|}{ Revenus } & Montant en livres \\
\hline Les gages annuels & 1500 livres \\
\hline Les épices bi-annuels & $\begin{array}{r}\text { Entre 251 livres et 376 livres } \\
\text { selon les séances et le nombre } \\
\text { d'affaires traitées }\end{array}$ \\
\hline Les menues nécessités annuelles & 39 livres \\
\hline Les droits de bonnet & 6 livres et 8 sols pour tout nouvel \\
& arrivant à la Chambre \\
\hline Le droit de traite annuel & 6 livres \\
\hline Total & Entre 1800 et 1950 livres \\
\hline
\end{tabular}

\section{La vente de l'office}

Au bout de vingt ans, Salomon décide de vendre son office, et il décrit minutieusement le détail de la vente dans son dial. Le 8 juillet 1656, Salomon vend à Guillaume Arthur, sieur de la Motte, pour 75500 livres, la charge de maître ordinaire à la Chambre des comptes qu'il avait acheté 47000 livres vingt ans auparavant. Le bénéfice réalisé, de près d'un tiers par rapport au prix d'origine, est loin d'être négligeable. De fait, cette vente représente l'essentiel du profit que l'on puisse tirer de l'office. Les modalités de paiement sont fixées dans le contrat de vente, et le premier paragraphe stipule que

49. R. MOUSNIER, op. cit., p. 78.

50. Arch. dép. de Loire-Atlantique, série B : mandements royaux.

51. Ce tableau a été réalisé d'après les livres de raison de Salomon (Arch. dép. de LoireAtlantique, 2 E 1383/12) et le Papier dial des revenus de son office de maître aux Comptes (idem, 2 E 1383/19). 
l'acquéreur " payera content ${ }^{52}$ la somme de cinq cents livres, et le surplus montant soixante et quinze mil livres, les payera audit sieur de La Tullaye ou à son ordre en cette ville de Nantes, quitte en sa main ou à son domicile $^{53}$ dans six ans prochains ${ }^{54}$ ", et " jusques au parfaict payement dicelle somme, il payera les intérêts à raison du deniers saize par les demies années $^{55}$ ". Rien n'est laissé au hasard, et cela se justifie par l'importance de la somme en jeu. De plus, Salomon exige qu'aucun versement « ne pourra estre moindre de quatre mil huict cent livres ", car les intérêts sont calculés sur un mode dégressif, en fonction de chaque paiement, et diminuent au fur et à mesure que l'acquéreur rembourse le vendeur. Salomon se montre beaucoup plus intransigeant envers le sieur de la Motte qu'on ne le fut avec lui-même lorsqu'il acheta son office. Les remboursements dépendaient alors de lui, et non d'un contrat aussi strict. De plus, le deuxième paragraphe du contrat indique que « ledit sieur Arthur n'entrera en la jouissance des droicts dudit office que du premier jour de janvier prochain, jusques auquel jour ledit sieur de La Tullaye se réserve tous et chacun les droicts, gages et esmoluements de ladicte charges escheus et à escheoir jusques

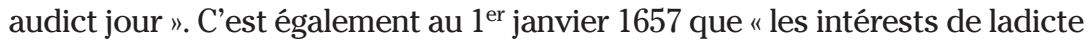
somme de soixante et quinze mil livres commenceront à courir ${ }^{56}$ ".

Les remboursements du sieur de la Motte sont soigneusement consignés dans le dial des affaires domestiques de Salomon comme dans celui de son office ${ }^{57}$, suivant cette habitude qu'a le sieur de La Tullaye de tout noter en plusieurs exemplaires. Ces remboursements s'avèrent relativement resserrés dans le temps, puisqu'à la fin de l'année 1659, Guillaume Arthur ne doit plus que 24729 livres et 3 sols " de reste du prix principal et les intérests de ladicte somme depuis le premier juillet 1659 ». L'acquéreur s'est donc montré bon payeur, ayant acquitté près des deux tiers de sa dette en moins de trois ans. La quasi intégralité de ces sommes va servir à Salomon dans l'engagement de nombreux contrats de constitution, également consignés dans son dial de maître aux comptes ${ }^{58}$. Dans ce type de contrat, Salomon prête de l'argent à une personne, à charge pour cette dernière de lui verser régulièrement des intérêts sous forme de rente - généralement un dixième de la somme principale - jusqu'au remboursement intégral et en une seule fois de l'argent prêté. D'après son dial, on observe

52. Salomon de La Tullaye écrit systématiquement " content " pour " comptant ". C'est là une des rares fautes d'orthographe flagrantes que l'on peut relever dans ses écrits.

53. Arch. dép. de Loire-Atlantique, 4 E 2/179 : Contrat de vente de l'office. Ceci est d'autant plus facile que les deux hommes sont voisins, et habitent tous deux la paroisse NotreDame de Nantes; Salomon " en sa maison de la Suze " et " le sieur de la Motte en la maison où est demeurant noble homme Pierre Courtois, dicte parroisse de Notre-Dame ".

54. Ibidem.

55. Ibid.

56. Ibid.

57. Idem, 2 E 1383/17 : "Papier dial du revenu de mon office de maistre des Comptes ", du côté des " deniers provenant de la vente que j'ay faicte au sieur de la Motte de mon office de maistre des Comptes, ensemble le remploy desdicts deniers".

58. Ibidem. 
que Salomon, en homme prudent et avisé, prête peu de grosses sommes, et préfère multiplier les petites transactions avec divers partenaires ${ }^{59}$. La fin de cette partie du dial en 1665 suggère qu'à cette date le sieur de la Motte ait intégralement remboursé le prix de l'office, en moins de dix années. La transaction ne s'arrête pourtant pas là, et Salomon ne réclame pas seulement de l'argent en contrepartie de son office.

\section{Les lettres de maître honoraire}

La suite du contrat de vente de l'office stipule que « ledict sieur Artur fournira à ses fraiz audict sieur de La Tullaye, à sondict domicile, dans les deux moys prochains, des lettres d'honoraires soubs le nom dudict sieur de La Tullaye, qui, pour cet effect, lui fournira coppie de l'arrest de sa réception audict office, pour iustifier qu'il a servi vingt ans parfaicts et accomplis $^{60}$ ". Faut-il voir dans cette clause une preuve de l'attachement de Salomon au travail qu'il a fait pendant vingt ans? Ce n'est pas impossible, vu le goût du personnage pour les comptes rigoureux, tant dans ses affaires privées, que dans le cadre de la Chambre. Quoi qu'il en soit, la raison de ces lettres demandées par Salomon ne saurait être d'ordre financier. En effet, les lettres patentes que Salomon reçoit le 8 septembre 1656 spécifient bien que s'il pourra jouir " des mesmes honneurs, authorités, franchises, libertéz et immunités appartenant audict office en la mesme manière que les autres officiers honoraires en jouissent en ladicte Chambre ", il ne pourra néanmoins " prétendre aucuns gaiges, droicts ni aultres proffictz et esmoluements $^{61}$ ». Ces lettres reconnaissent en contre partie à Salomon sa " singulière prudence et affection au bien de nostre estat, qui méritte une recognoissance particulière ", et " aussy qu'il n'est pas raisonnable que l'expérience qu'il c'est acquise dans l'exercice dudict office [...] demeure infructueuse, c'est pourquoi nous avons estimé juste et utille à nous et au public de tesmoigner audit de La Tullaye la satisfaction que nous avons de ses services, en le favorisant des marques d'honneur que nous avons accoustumé de départir à ceux quy, par un si long temps, se sont dignement acquittés de leurs charges ". La suite de la lettre ne peut que flatter Salomon, en espérant " que cet exemple excite nos améz officiers à faire le semblable".

Le travail de Salomon, sa rigueur et sa probité sont donc reconnus officiellement par l'institution, et il apparaît que c'est avant tout le goût des comptes et du travail bien fait qui l'ont guidé tout au long de sa carrière. Le même souci comptable s'observe de la même manière dans sa vie privée, comme le prouve son dial de ses affaires domestiques. Celui-ci révèle, à l'insu de son auteur, des aspects de la vie quotidienne d'un membre de la moyenne noblesse durant le Grand Siècle.

59. Ibid., Salomon prête 2000 livres à Bernard de Poulpiquet, président de la Chambre des comptes - et donc a priori solvable - la même somme à Guillaume Raoul, également président aux Comptes, mais aussi 2400 livres à Geoffroy Rohée, marchand.

60. Ibid.

61. Idem, registre B $81, \mathrm{f}^{\circ} 59$. 


\section{La vie quotidienne chez Salomon de La Tullaye à travers ses livres de raison}

\section{Les dépenses quotidiennes}

Son livre de raison est pour Salomon de La Tullaye un moyen quasi infaillible de faire ses comptes. Chaque petit cahier, composé de feuilles pliées en huit, et cousues en leur centre, est divisé en deux parties : l'une pour les dépenses; l'autre pour les recettes ${ }^{62}$. Chaque créancier ou débiteur est nommé avec précision, ainsi que son lieu d'habitation et la ou les sommes dues ou perçues. Seuls deux cahiers manquent, perdus au cours des siècles.

Les recettes proviennent essentiellement des métairies et borderies de Salomon, puis de son office de maître aux comptes, et enfin des divers contrats de constitution qu'il a engagés et qui lui rapportent des rentes régulières. Les dépenses sont relatives aux mille et une choses du quotidien, telles que les réparations inévitables dans une vieille demeure, la nourrice des enfants - Salomon aura six enfants de ses deux mariages mais aussi l'entretien du carrosse, les achats et façons de vêtements, ou encore les gages des serviteurs. Une grande partie des dépenses concerne bien évidemment la nourriture, mais le détail n'en est pas précisé. Pour l'année 1642, Salomon calcule que du 11 septembre 1641 au 11 septembre 1642, " la dépence de bouche de [s] a maison, en ce compris le boys, la chandelle, vin d'Anjou, pain, viande et autre menue despence de cuisine, et le tout pour ladicte année monte à 2177 livres, 10 sols et 10 deniers, en ce non compris le vin nantais pour les serviteurs, ny foing ni avene ${ }^{63}$ pour les chevaux $^{64}$ ". Cette somme engloutit à elle seule les revenus que Salomon tire de sa charge de maître à la Chambre des comptes.

Un tableau des dépenses et recettes de l'année 1640 (tableau n ${ }^{\circ}$ 3) a pu être réalisé à partir du dial de Salomon, dans lequel il apparaît que le sieur de La Tullaye vit légèrement au-dessus de ses moyens, dépensant près de 12000 livres, quand il n'en rentre que 9000 dans son coffre. Le déficit tourne donc autour de 3000 livres, mais peut être résorbé les années suivantes, car certaines dépenses ne reviennent pas chaque année. Avec plus de 2000 livres, la dépense de bouche de la maison de Salomon représente par conséquent un cinquième à un quart de son budget. Le manque de détails de ce type dans les livres de raison des années suivantes empêche d'établir une comparaison, et l'on ignore si les dépenses excèdent toujours les recettes. De plus, Salomon multiplie les comptes avec sa famille et sa bellefamille, certains parents réglant pour lui des factures pour éviter de se déplacer. Il va sans dire que Salomon leur rend la pareille. Les comptes sont difficilement comparables à cause de cet échange de bons procédés. Apparaît ensuite du côté des dépenses tout ce qui concerne l'habillement.

62. Cf. figure $\mathrm{n}^{\circ} 2$.

63. Salomon écrit toujours " avène " pour avoine.

64. Arch. dép. de Loire-Atlantique, 2 E 1383/12 : Livre de raison. 
Austère, il n'en est pas moins cossu. Salomon affectionne les habits de drap de Hollande, de couleur sombre - obéissant en ceci à la tradition vestimentaire des officiers et gens de justice - doublés de taffetas, et agrémentés de plusieurs douzaines de boutons ${ }^{65}$, le tout pour 67 livres.

\section{Tableau $\mathrm{n}^{\circ} 3$ - Tableau général des dépenses et recettes de Salomon de La Tullaye pour l'année 1640}

\begin{tabular}{|l|c|c|}
\hline \multicolumn{1}{|c|}{ Mois } & Dépenses & Recettes \\
\hline Janvier & 627 livres et 10 sols & 1450 livres \\
Février & 532 livres et 1 sol & 669 livres et 2 sols \\
Mars & 3095 livres & 68 livres \\
Avril & 504 livres et 10 sols & \\
Mai & 594 livres, 15 sols et 1 denier & 263 livres \\
Juin & 394 livres, 12 sols et 6 deniers & \\
Juillet & 1035 livres, 13 sols et 7 deniers & 1550 livres \\
Août & 1545 livres 6 sols et 1 denier & 929 livres et 3 sols \\
Septembre & 280 livres & 3356 livres et 5 sols \\
Octobre & 68 livres et 12 sols & \\
Novembre & 2256 livres et 5 sols & 287 livres, 18 sols et 9 deniers \\
Décembre & 1056 livres, 7 sols et 2 d. & 627 livres et 10 sols \\
\hline \multicolumn{1}{|c|}{ Total } & 11990 livres et 11 sols & 9200 livres et 19 sols \\
\hline
\end{tabular}

Peu d'achats de bas sont mentionnés, sinon des « bas d'Angleterre de laine noire "; il semble que Salomon leur préfère les " calcons, chaussettes et chaussons de peau de chamois ${ }^{66}$ ". Quant aux chaussures, ce sont des " bottines de marroquin noir ${ }^{67}$ " ou des souliers, mais la couleur ni la matière n'en sont précisées. On sait seulement qu'en 1646, une paire achetée au cordonnier de Richebourg coûte 58 sols, contre 40 sols pour la paire que Salomon fournit à son laquais.

Le maître doit en effet nourrir et habiller ses gens. Celui qui lui coûte le plus cher est son unique laquais, lequel représente en quelque sorte son maître, et se doit de porter sur lui un peu de son apparat vestimentaire. En 1646 , Salomon lui achète un habit de " sarge $^{68}$ " grise, doublée de jaune, le tout pour 20 livres, souliers compris. La domesticité tient une place importante dans les livres de raison, pour les dépenses vestimentaires et l'entretien - tous les domestiques sont nourris et logés - mais surtout pour les

65. Ibidem : note du 6 novembre 1646 : " J'ay payé à Massonneau, marchand de soye, 7 livres, 17 sols et 7 deniers, pour une aulne trois quarts de taffetas pour doubler mon pourpoint de drap de Hollande, et j'ay payé à Me Gouzeau bouttonier 4 francs pour saize douzaines de bouttons pour mon habit de drap de Hollande. "

66. Ibid., note du 16 décembre 1662 : "J'ay payé à un marchant d'entre les portes de Sainct Nicolas 12 livres et 15 sols pour troys peaux de chamois, pour faire une paire de calcons, chaussettes et chaussons de chamois. " La peau de chamois est de la peau de mouton.

67. Ibid., note du 6 décembre 1644 .

68. Ibid., note du 27 novembre 1646. La " sarge " est sans doute de la serge. 
gages annuels. Ceux-ci n'ont que très peu augmenté pendant les trente années suivantes.

Tableau n ${ }^{\circ} 4$ - Domesticité de Salomon de La Tullaye pour l'année 1640

\begin{tabular}{|l|l|}
\hline \multicolumn{1}{|c|}{ Fonction } & \multicolumn{1}{c|}{ Gages annuels } \\
\hline 1 laquais & sans doute plus de 60 livres \\
1 valet de chambre pour Salomon & de à 50 livres \\
1 femme de chambre & 14 écus, soit à peine 45 livres \\
pour Madame de La Tullaye & \\
1 femme de chambre pour les petites filles & 16 écus, soit près de 50 livres \\
1 servante de cuisine & 30 livres \\
1 palefrenier & 36 livres \\
1 cocher & 20 écus, soit 60 livres \\
1 jardinier au Plessis-Tison & 16 écus, soit près de 50 livres \\
1 jardinier à la Suze & Sans doute les mêmes gages \\
& que celui du Plessis-Tison \\
\hline \multicolumn{1}{|c|}{ Total } & \multicolumn{1}{|c|}{432 livres } \\
\hline
\end{tabular}

La maison de Salomon se constitue ordinairement d'une dizaine de domestiques, tous spécialisés. Deux personnes préposées au transport (par carrosse) et deux jardiniers (car il y a deux propriétés) indiquent un train de vie de noblesse aisée. Si l'on prend comme exemple l'année 1640 durant laquelle Salomon de La Tullaye dépense 11990 livres, soit presque 12000 livres (tableau $\mathrm{n}^{\circ}$ ) les gages de ses domestiques, qui sont de 432 livres par an correspondent à environ un trentième du budget annuel. À titre de comparaison avec les familles nobles bretonnes étudiées par Jean Meyer ${ }^{69}$, seules $18 \%$ de ces familles ont plus de cinq domestiques, tandis que la grande majorité se contente d'une à deux personnes. Avec dix domestiques, Salomon de La Tullaye se situe dans la fraction aisée de la noblesse. Il ne peut cependant prétendre appartenir à la grande noblesse; en effet, quand il donne 45 livres par an à son jardinier en 1658, Madame de Sévigné, elle, en offre 140 au sien ${ }^{70}$.

Bien que nourris et logés, les gens de Salomon ne restent cependant guère en place. Pas moins de dix laquais se succèdent en une vingtaine d'années. L'un d'eux, "Louys, dict Esprit " est même congédié en mars 1640 , au bout de huit mois de services ${ }^{71}$. Le roulement régulier de la domesticité peut être imputé à un service pénible dans un hôtel compor-

69. Jean Meyer, La noblesse bretonne au XVIII siècle, Bibliothèque de l'École Pratique des Hautes Études VI ${ }^{\mathrm{e}}$ section, SEPVEN, Paris, 1966, p. 1190. Les recherches de M. Meyer sont basées sur la capitation de 1710, soit quelques dizaines d'années après Salomon, mais on peut accepter ces chiffres pour la fin du XVII ${ }^{\mathrm{e}}$ siècle, car ils n'ont dû que peu évoluer.

70. Ibid., p. 1193.

71. Idem, 2 E 1383/12 : livre de raison, note du 8 mars 1640. La plupart des domestiques de Salomon entre 1637 et 1640 portent un surnom, tel "Louys dict Esprit ", "Poirier dict La Forest " ou encore "Vincent dict la Chesnaye ", sans que l'on en sache la cause ni l'origine. 
tant trois à quatre étages, et Salomon, comme la plupart des maîtres, ne paye les gages qu'au bout de longs mois. Ainsi Joseph, le cocher qui conduit son carrosse, entré à son service le 25 janvier 1645, ne touche-til ses gages qu'au mois de décembre suivant, et encore n'en perçoit-il qu'un tiers, soit 15 livres, pour des appointements de 60 livres par an. C'est d'ailleurs le mieux payé des gens de la maison. À l'opposé, au bas de la hiérarchie ancillaire, se trouve la servante de cuisine, qui travaille pour la modique somme de 30 livres l'année. Pas de cuisinier permanent à la Suze; Salomon en fait venir un, appelé " Bonnestienne " spécialement pour les grandes occasions, notamment pour " un disner auquel [il] traictait Messieurs de la Chambre " en juillet $1640^{72}$. Les gens de Salomon, s'ils ne touchent que tardivement leurs gages, perçoivent tout de même de petites avances tout au long de l'année. En décembre 1640, ayant donné 60 sols à son valet Baptiste Guibert, Salomon s'étonne dans son dial de l'usage que l'homme en a fait : " il a achepté une guytarre ${ }^{73}$ ". La dépense semble sans doute parfaitement incongrue aux yeux de Salomon pour qu'il prenne ainsi la peine de la noter.

Les domestiques sont régulièrement présents dans les comptes de Salomon, mais il ne faut pas en oublier pour autant que le véritable entourage du sieur de La Tullaye est sa famille. C'est en pointillés qu'elle apparaît, derrière les chiffres de ce comptable rigoureux.

\section{Salomon et ses épouses}

En 1636, son premier mariage n'inspire à Salomon qu'un laconique « j'ay fiancé Mlle des Briottières par contract passé ledit jour 18 febvrier 1636, passé par Quenille notaire à Nantes ", puis, quelques semaines plus tard : "Le 24 Apvril, j'ay espousé Renée de Lesrat en l'église Nostre Dame de Nantes, par l'archevêque Fourché ${ }^{74}$. " Bon prince, Salomon concède à sa jeune épouse - elle a vingt-deux ans - " quatre violons pour [s]es nopces". Dès lors, la nouvelle Madame de La Tullaye quitte la tutelle paternelle pour passer sous celle de son époux. Renée apporte une belle dot de 27000 livres, qui tombe à point pour financer l'office de maître des comptes que brigue Salomon. Malgré cela, la jeune femme n'a jamais accès à la bourse du ménage, et son époux lui verse chaque trimestre une somme de 75 livres pour son entretien ${ }^{75}$. C'est également en 1636 que Salomon se trouve tuteur de ses deux jeunes petits-cousins, dont l'aîné, René, est presque de l'âge de la jeune Madame de La Tullaye, puisqu'il a dix-huit ans. Il semblerait que Salomon, âgé de trente-sept ans, ait englobé dans une même attitude paternaliste sa jeune épouse et ses petits-cousins de Belle

72. Ibidem, note du 30 juillet 1640 .

73. Ibid., note du 13 décembre 1640.

74. Ibid., notes du 18 février et du 24 avril 1636.

75. Ibid., note du $1^{\text {er }}$ juillet 1639 : « J'ay payé à ma femme 75 livres pour son entretien pendant troys moys qui finiront le $1^{\text {er }}$ octobre 1639 ." 
Isle ${ }^{76}$. Renée de Lesrat vient pourtant d'une famille de conseillers au Parlement, et cette alliance s'est avérée fort utile à Salomon lors de l'adjudication de son office de maître des comptes. De son mariage avec Salomon, Renée a trois filles : Françoise en 1637, Marquise en 1638 et Anne en 1639. Quelques années plus tard, le 7 décembre 1643, Renée meurt à trente-neuf ans " grosse d'une fille dont elle ne peut accoucher ${ }^{77}$ ". Salomon ne livre dans son dial aucun sentiment personnel et ne s'autorise aucune marque de chagrin au décès de celle qui a partagé sa vie près de dix ans. Veuf avec trois filles, il ne tarde pas à convoler de nouveau, avec une autre angevine, Françoise Martineau, en décembre 1644.

La nouvelle épouse, qui n'a que vingt-trois ans, est déjà veuve de Mathieu de la Lande, prévôt d'Anjou. La famille Martineau a donné deux maires et des juges prévôts à la ville d'Angers, et cette nouvelle alliance est à nouveau profitable à Salomon. La différence d'âge entre les deux conjoints est encore plus criante que lors du premier mariage de Salomon, puisqu'il a alors quarante-cinq ans. Pourtant, dès le début de ce mariage, on observe que Françoise est beaucoup plus indépendante financièrement que ne l'était Renée de Lesrat. Elle règle elle-même les créanciers, et passe régulièrement commande chez les marchands ${ }^{78}$. Au milieu des années 1640, Salomon a trois petites filles à élever, et se partage entre son foyer et sa charge de maître des comptes, tout en continuant à gérer les affaires de ses petits-cousins de Belle Isle. On peut raisonnablement penser qu'il se repose alors un peu plus sur son épouse pour les dépenses de la vie quotidienne, principalement celles qui touchent au domaine vestimentaire, tant pour elle-même que pour ses enfants. Françoise va donner à Salomon trois enfants également : Jeanne en 1648, Élisabeth en 1650, et SalomonFrançois en 1653. Salomon se retrouve ainsi père de six enfants et note scrupuleusement ce que lui coûte leur entretien.

\section{Salomon et ses enfants}

Il semble que le maître de maison laisse à son épouse le soin des enfants, et, dans la mesure où les cinq premiers sont des filles, il est logique que ce soit Madame de La Tullaye - il s'agit de Françoise - plutôt que Monsieur, qui passe commande chez le marchand de soie ${ }^{79}$. Ceci n'est qu'un exemple parmi d'autres illustrant la mentalité de Salomon quant aux enfants en général, et à sa progéniture en particulier : c'est l'affaire des femmes et non point des hommes. Le maître n'intervient que pour payer la sage-femme, et noter dans son livre de raison l'heure de naissance et la

76. Le jeune René de La Tullaye a hérité de son défunt père de la seigneurie de Belle Isle, à Nantes sur les bords de l'Erdre.

77. Arch. dép. de Loire-Atlantique, 2 E 1383/3.

78. Idem, 2 E 1383/12 : livre de raison, note du 18 juillet 1646 : « Ma femme a payé au sieur de Lespine 30 livres pour apprendre à danser à mes filles Fracoyse et Marquise. "

79. Ibidem, note du 10 décembre 1652 : « J'ay payé à Mr Baber 166 livres pour la levée de deux robbes de tabiz changeant avec dentelles or et argent et autres menues fournitures. " 
date du baptême de l'enfant, conformément à d'autres livres de raison de la même époque. Ce n'est, dit Madeleine Foisil, que « la retransmission de la vie qui est consignée ${ }^{80} "$.

Salomon et Françoise perdent deux enfants, dont un fils qui n'a vécu que quelques jours. Rien dans les notes ne vient souligner la douleur du père (ou de la mère, mas celle-ci n'apparaît pas dans une écriture ici uniquement masculine), et seul le terme de " petit enfant ${ }^{81}$ " échappe à Salomon au moment d'écrire les frais de l'enterrement. On est bien loin de Charles Demaillasson, petit notable poitevin et contemporain de Salomon, qui rappelle avec émotion dans son journal les qualités de ses petits-fils morts. Il est vrai, comme le remarque Madeleine Foisil, que " le livre de raison, qui est livre de comptes, n'est point propre à l'épanchement du chagrin et du deuil ${ }^{82}$ ". Salomon suit cette définition avec une rigoureuse exactitude. La perte d'un enfant est le lot commun, et l'on s'estime encore assez heureux quand la mère ne décède pas en couches. Une particularité, imputable à une santé fragile, marque les enfants de Salomon : ils sont tous ondoyés $^{83}$ au logis. Après la naissance, les enfants sont immédiatement confiés à une nourrice. Seules les femmes du commun allaitent, en France comme en Angleterre, rappelle Élisabeth Bourcier dans son travail sur les journaux anglais ${ }^{84}$. Chez les La Tullaye, l'enfant reste généralement un an et demi à deux ans chez chaque nourrice. Celle-ci est souvent la femme d'un métayer, comme Françoise Arnoul, nourrice des deux cadettes du premier mariage de Salomon, épouse d'Hervé Garnier, dit de l'Isle, bordier de La Guilbaudière ${ }^{85}$. Après la nourrice, les fillettes, âgées d'une dizaine d'années, partent en pensionnat religieux. Les deux aînées, Françoise et Marquise, vont chez les religieuses de Sainte-Élisabeth ${ }^{86}$ à Nantes, la petite Anne au couvent de la Visitation à Angers - où elle prononcera plus tard ses vœux - et Jeanne chez les ursulines ${ }^{87}$ de Nantes. Auparavant, elles ont appris à danser avec le sieur de l'Espine, et la dame Périer leur a fait « l'escolle $^{88}$ ", afin de pouvoir, plus tard, tenir leur rang dans la bonne société

80. Madeleine FoIsIL, "L'écriture du for privée ", Histoire de la vie privée, dir. par Ph. ArIEs et G. DuBY, Seuil, Point Histoire, 1985, 1999, t. 3, p. 338.

81. Arch. dép. de Loire-Atlantique, 2 E 1383/12 : "Le 25 février 1647, ma femme accoucha que les 8 heures du soir de son second enfnat qui est un garçon, lequel a esté baptisé au logis et le $1^{\mathrm{er}}$ jour de mars ensuivant, sur les 6 heures du matin, edit enfant décéda et fut enterré le même jour dans mon enfeu [...] église Nostre Dame ", et note du $1^{\mathrm{er}}$ mars 1647 : "Payé au vicaire de Nostre Dame 4 francs pour 10 messes et 20 sols pour la messe et service du petit enfant."

82. M. FoIslL, "L'écriture du for privé ", op. cit., p. 339.

83. C'est-à-dire baptisés par un laïc à la maison, quand l'enfant n'est pas en bonne santé et risque de ne pas survivre jusqu'au baptême.

84. Élisabeth BourciER, Les journaux privés en Angleterre de 1600 à 1660, Publications de la Sorbonne, Imprimerie Nationale, Paris, 1976, p. 212.

85. Arch. dép. de Loire-Atlantique, 2 E 1383/12 : livre de raison, note du 19 janvier 1640. 86. Idem, 2 E 1383/2 : livre de raison, quittances du pensionnat de Sainte-Élisabeth pour les années 1647-1650.

87. Id., 2 E 1383/12 : livre de raison, notes du 22 avril 1660.

88. Ibidem, notes des 18 juillet et 10 octobre 1646 . 
nantaise. Salomon, par ailleurs si sec dans ses livres de raison, prend l'habitude de nommer ses enfants par un surnom affectueux. Ainsi Françoise devient-elle "Francillon ", Anne " Annette " ou Jeanne " Jeanneton ». Quant au petit dernier, le fils sans doute très attendu, nommé des deux prénoms de ses parents, Salomon-François, il ne va pas en pension, mais reçoit l'enseignement d'un précepteur en la personne du curé de la paroisse Saint Léonard de Nantes ${ }^{89}$.

Cinq filles représentent cinq dots à payer, même si elles deviennent des religieuses. Le contrat de religion d'Anne, dix-neuf ans, au couvent de la Visitation d'Angers, date du 30 octobre 1658 et stipule que le montant de la dot s'élève à 9000 livres $^{90}$. La dot de sa demi-sœur Élisabeth, quinze ans, qui entre au même couvent, le 30 juin 1665, est moins élevée : 6500 livres seulement, sans qu'en soit précisée la raison ${ }^{91}$. Il est à noter que dans chacun des deux mariages de Salomon, c'est toujours la dernière fille qui entre au couvent. L'aînée de chaque union, elle, est mariée à un bon parti. Dans le cas d'Anne, la jeune fille a visiblement changé d'avis, car Salomon écrit le 3 juin 1656 que «n'ayant dessein d'estre religieuse ${ }^{92}$ ", Anne quitte le couvent et va " en pension à Angers chez une dame nommé Gratienne Bontier, sœur du Mr Tonnelier, prestre chapelain des Filles de la Visitation d'Angers $^{93}$ ". Cette phrase indique que Salomon n'exerce pas de pression sur sa fille pour la faire entrer en religion. Pourtant, Anne retourne à la Visitation pour y prononcer ses "vœux de pauvreté, chasteté et obédience $^{94}$ " le 23 novembre 1659, sans que Salomon n'explique dans son dial la cause de ce revirement.

En 1667, Salomon fait épouser à Françoise, sa fille aînée, " Messire Louis Binet, chevalier, sieur de la Belotière ${ }^{95}$, fils de deffunt Messire Victor Binet, seigneur de Montifroy, conseiller du Roy en ses conseils, et Président de la Chambre des comptes de Bretagne, de son mariage avec dame Jeanne du Pé, fille de Messire Charles du Pé, seigneur d'Orvault et de dame Marquise de la Porte, ladite Jeanne du Pé à présent femme de Messire Jean Fourché, seigneur de Quéhillac, conseiller du Roy en se conseils et procureur général sindic des Estats de Bretagne ${ }^{96} "$. Salomon signe les articles du contrat de mariage le 5 février, et le mariage a lieu trois mois plus tard, le 17 mai, dans la chapelle du château du Plessis-Tison. Les 46000 livres de dot apportée par la mariée peuvent compenser son âge - elle a trente ans - et se composent de métairies, marais salants et divers contrats de constitution, ainsi que des " habits de nopces ${ }^{97}$ ". Ces derniers coûtent d'ailleurs mille livres

89. Ibid., note du 22 mars 1663.

90. Idem, 2 E 1383/12 : note du 30 octobre 1658.

91. Id., 2 E 1383/2.

92. Id., 2 E $1383 / 5$.

93. Id., 2 E 1383/12: note des 2 et 3 juin 1656.

94. Ibidem, note du 23 novembre 1659 .

95. Il s'agit de La Blottière.

96. Arch. dép. de Loire-Atlantique, 2 E 1383/12 : note du 5 février 1667.

97. Ibidem, note du 17 mai 1667. 
à Salomon, et la somme prouve qu'il ne s'agit pas ici d'un petit mariage! L'aînée du second mariage de Salomon, Jeanne, est mariée à Henri du Bois, sieur de la Ferronnière, mais on n'a que fort peu d'indications sur ces noces. En revanche, Salomon s'emploie à organiser au mieux le mariage de son seul fils, Salomon-François. Il jette son dévolu sur la fille d'Yves Morice, sieur de Coetquelfen, procureur de la Chambre des comptes de Bretagne. Les deux hommes se connaissent déjà, ne serait-ce que par leurs fonctions dans la même institution, et aussi car Yves Morice a emprunté à Salomon la somme de 20000 livres tournois, en 1653. Le remboursement a lieu le jour du mariage de Marie-Anne Morice et de Salomon-François de La Tullaye, le 8 mai $1679^{98}$. Deux ans avant, dès le 9 septembre 1677, Yves Morice vend par contrat sa charge de procureur général à la Chambre des comptes à son futur gendre, pour une somme de 87000 livres "parce que ledit sieur de La Tullaye prendra pour femme damoiselle Marie Anne Morice, fille unique desdits sieur et dame de Coetquelfen, à laquelle il donne soixante trois mil livres en mariage ${ }^{99} "$. Au total, Salomon-François paye cette charge 150000 livres, ce qui correspond au prix payé par Morice en $1650^{100}$. Toute la vie que Salomon a passé à surveiller ses comptes, à lutter contre tout gaspillage, à engranger, denier après denier, une véritable petite fortune, trouve enfin son aboutissement dans le mariage de SalomonFrançois. Ayant pourvu toutes ses filles de dots, Salomon peut se permettre, sans l'emprunter, de déposer dans la corbeille de noces de son fils la coquette somme de 150000 livres. Bien des familles nobles de la province ne peuvent en faire autant. Salomon-François ne peut cependant entrer immédiatement à la Chambre, et doit vraisemblablement attendre le décès de son beau-père pour être reçu parmi ses pairs. Il reçoit ses lettres de provision le 29 mai 1682 et est reçu le 8 juin suivant ${ }^{101}$. Salomon, qui est resté vingt ans maître des comptes, permet à son fils d'accéder à l'échelon supérieur : l'office de procureur général. Dès lors, quatre générations de La Tullaye se passent le flambeau en ligne directe, jusqu'à la fin de la Chambre des comptes sous la Révolution.

"S'il est un besoin permanent, universel, lié à l'organisation même de l'esprit humain, que les intimistes ${ }^{102}$ éprouvent comme les autres hommes et qui les rend semblables à tous, c'est celui d'échapper à la fuite du temps, en gardant une trace écrite des instants fugitifs, et de conserver le souvenir de tout événement privilégié ", résume Alain Girard ${ }^{103}$. Salomon, en laissant derrière lui ses livres de raison, soigneusement rédigés et tenus à jour,

98. Idem, 2 E 1383/2 : acte de mariage entre Salomon-François de La Tullaye et MarieAnne Morice, le 8 mai 1679, sur velin.

99. Id., 2 E 1383/1 : répertoire d'actes.

100. Ibidem, Yves Morice acquiert la charge de procureur général par lettres du 9 janvier 1650, après résignation de René Le Prestre, pour la somme de 153000 livres.

101. Ibid.

102. L'intimiste est l'auteur d'un journal intime.

103. Alain GIRARD, Le journal intime, Bibliothèque de philosophie contemporaine, PUF, Paris, 1963, p. 7. 
n'a pas agi autrement. Certes, s'il conserve une trace écrite, c'est celle de ses rentrées d'argent ou de ses dépenses. Il a également eu toute sa vie le souci de conserver les vieux papiers de la famille, recopiant de vieux actes, dressant des arbres généalogiques qui se sont révélés extrêmement précieux pour étudier la famille de La Tullaye. Aucun papier ne fait état de ses sentiments, mais l'importante correspondance que Salomon entretient toute sa vie avec parents et amis pourrait bien être le lieu où il exprime ses sentiments. Malheureusement, le fonds d'archives ne recèle que la correspondance passive - c'est-à-dire reçue - de Salomon, et non la correspondance active. Le sieur de La Tullaye garde donc le secret de ses opinions et de ses émotions, et s'il nous en livre des fragments, au détour d'une ligne consacrée à ses enfants, c'est à son insu. Le personnage offre cependant un éclairage particulier - et donc précieux - de la vie quotidienne d'une famille de la noblesse nantaise, ni démesurément riche, ni très pauvre, une famille qui a progressé au sein de la notabilité locale sans à coup, mais sûrement et durablement. 


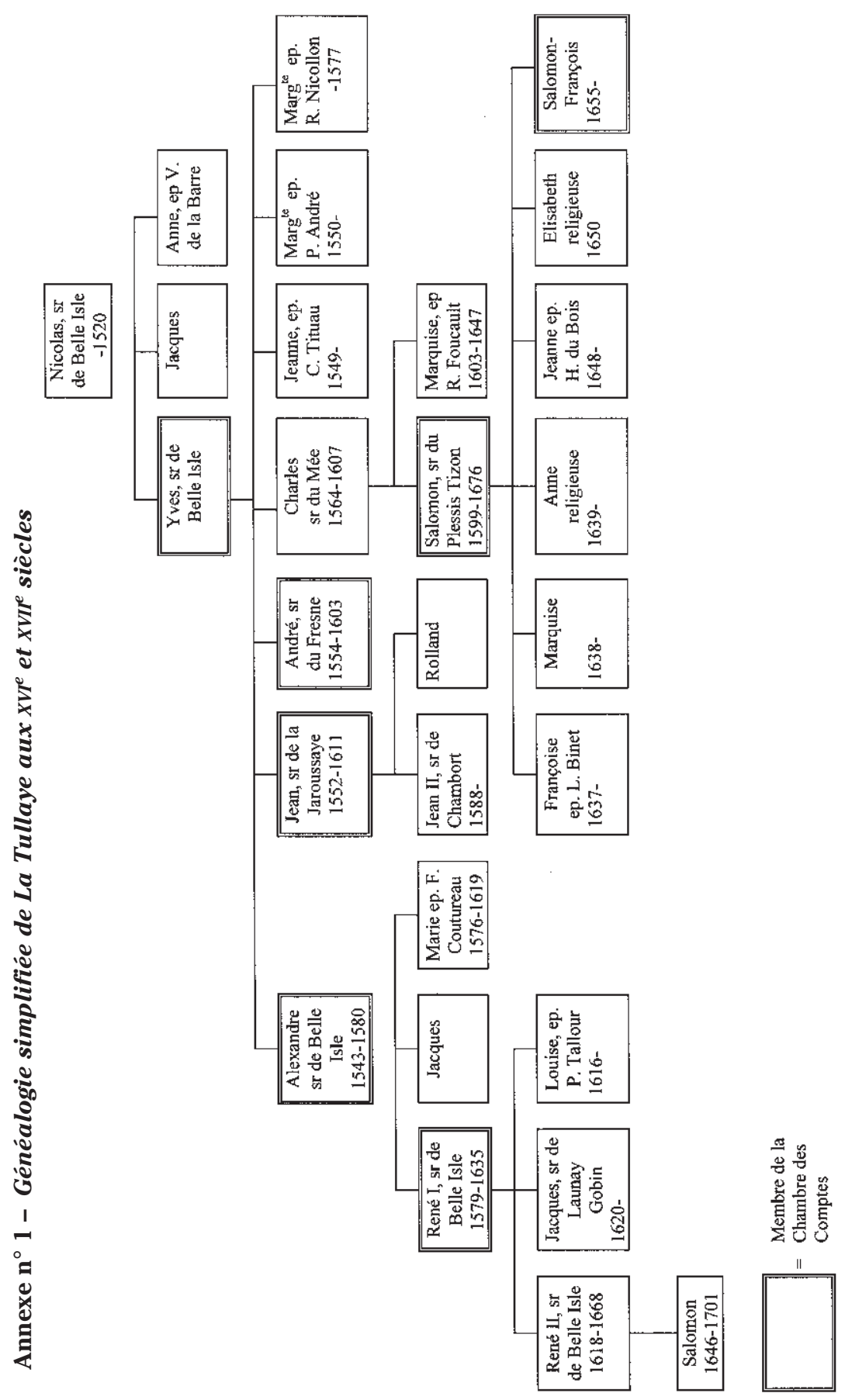


Éloïse MENARD

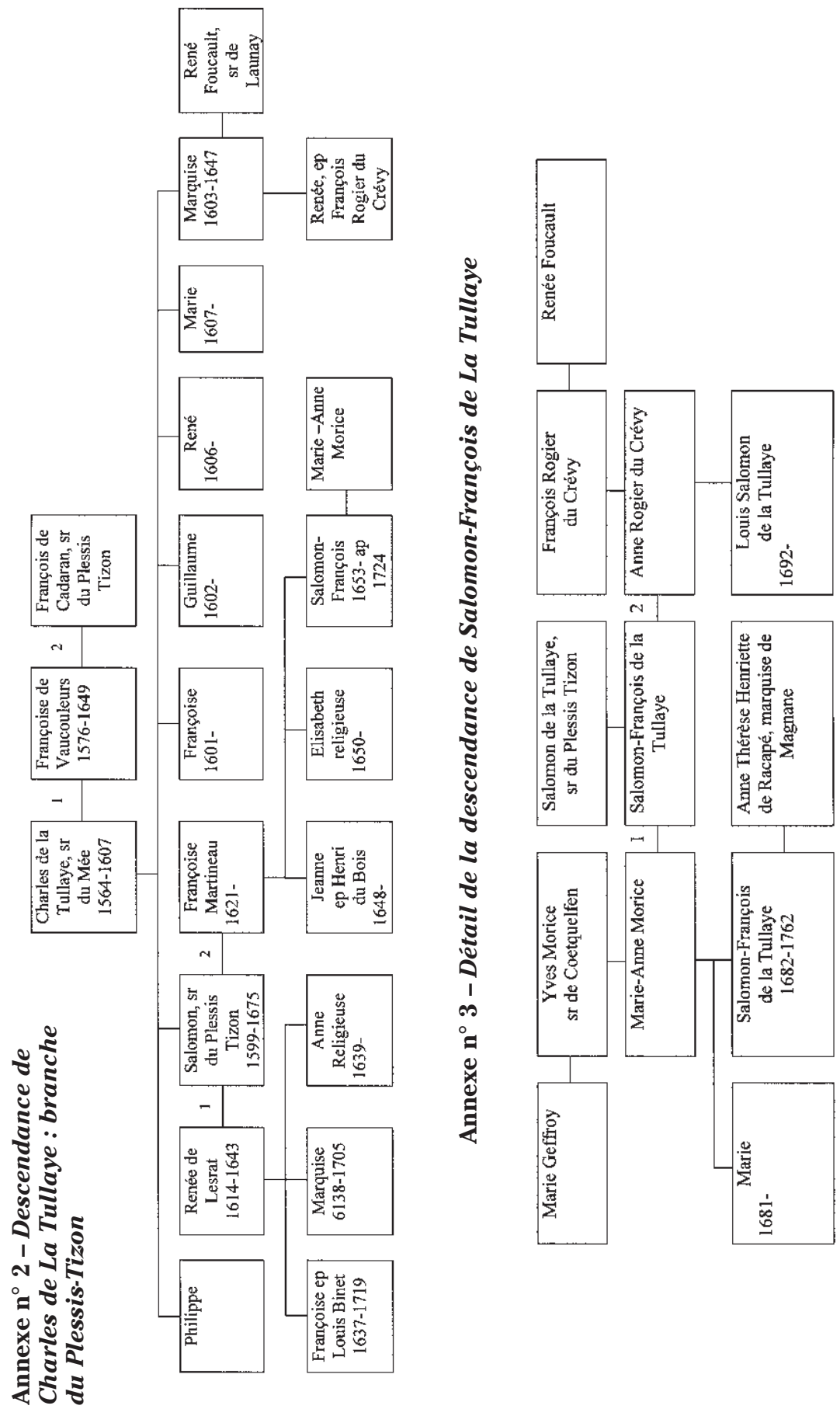




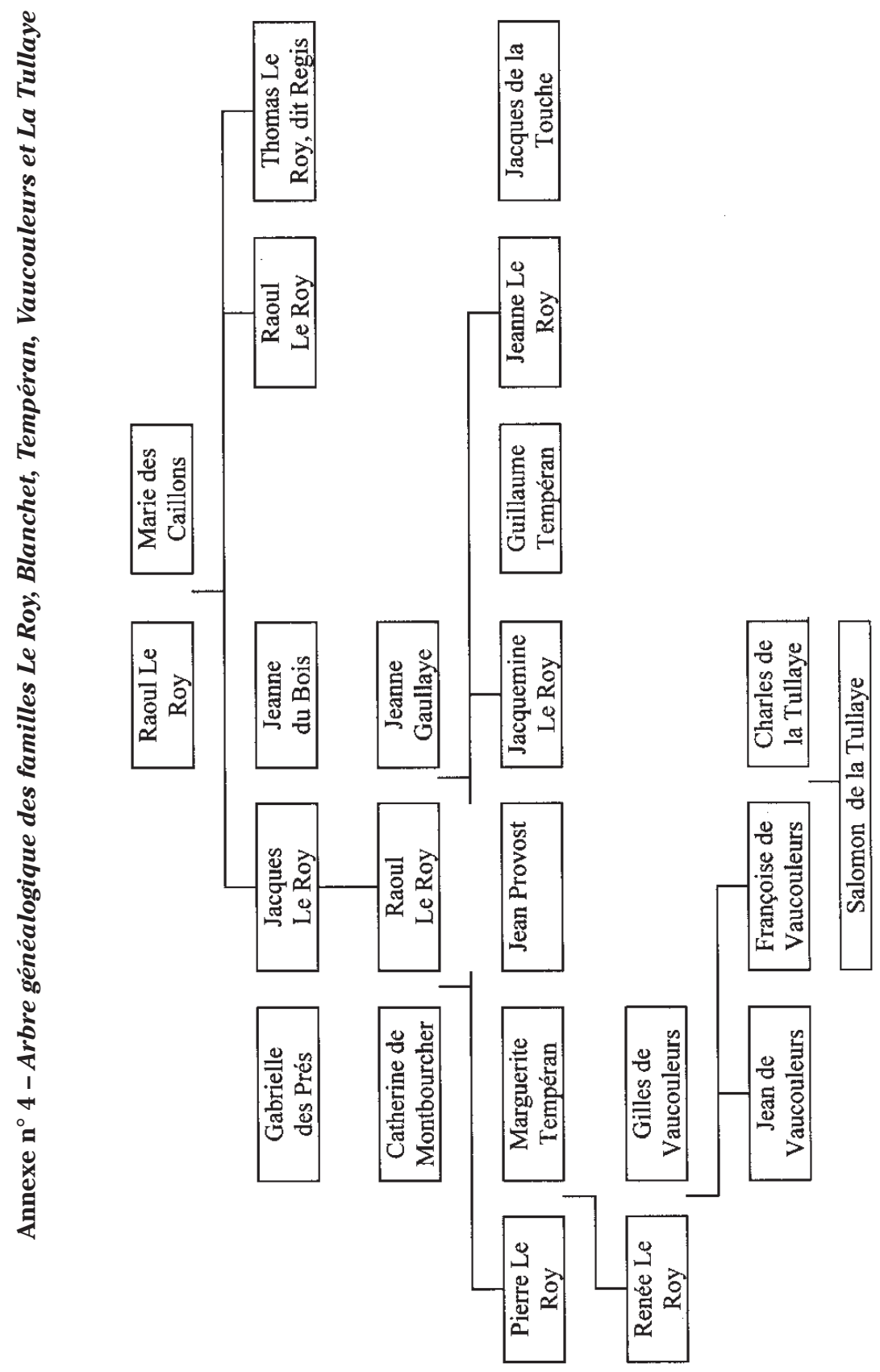


RESUME

Au XVII ${ }^{\mathrm{e}}$ siècle, Salomon de La Tullaye, appartenant à la noblesse nantaise, rédige au jour le jour un livre de raison. Ayant exercé pendant vingt ans la charge de maître aux comptes à la Chambre des comptes de Bretagne, Salomon de La Tullaye consigne soigneusement dans de petits cahiers ses activités ainsi que les gains relatifs à cet office. Il note également tout ce qui a trait à sa vie quotidienne, gages de ses valets ou leçons de musique de ses enfants. Toute sa vie apparaît ainsi en filigranes, derrière des listes de chiffres.

La famille de La Tullaye, petit lignage breton de la région de Fougères, s'est peu à peu hissée parmi les familles influentes de la municipalité nantaise. Salomon de La Tullaye est l'un des artisans de cette ascension, une sorte de personnage-charnière dans l'histoire de sa famille. Son fils Salomon-François de La Tullaye récolte ce que son père a patiemment semé pour lui, en devenant procureur général à la Chambre des comptes de Bretagne en 1682.

\section{ABSTRACT}

Salomon de La Tullaye [1599-1676] has written a diary for almost forty years, in which he has jotted down what he has earnt in his job in the Brittany Court of Accounts. He has written all his everyday life expenses too, the wages of his household for example. His diary is a very interesting one, even if there's no literary style inside.

Salomon de La Tullaye is a major figure in the history of his family. In the Middle Ages, this family was not a very famous nor a very strong one. Salomon was only a master of Accounts, but his son, Salomon-François, married the daughter of the public prosecutor in 1679. Afterwards, for four generations, the La Tullaye family members have been the public prosecutors. 
Achevé d'imprimer sur les presses du service reprographique de l'Université Rennes 2 Haute-Bretagne en décembre 2001 
